INFLAMMATORY BOWEL DISEASE

\title{
Anti-Saccharomyces cerevisiae antibody (ASCA) positivity is associated with increased risk for early surgery in Crohn's disease
}

\author{
D G Forcione, M J Rosen, J B Kisiel, B E Sands
}

Gut 2004;53:1117-1122. doi: 10.1136/gut.2003.030734

See end of article for authors' affiliations

Correspondence to: Dr B E Sands, Gastrointestinal Unit and Center for the Study of Inflammatory Bowel Disease, Massachusetts General Hospital, 55 Fruit St, GRJ 7, Boston, MA 02114, U'SA; bsands@ partners.org

Revised version received 15 January 2004 Accepted for publication 17 January 2004
Background: Anti-Saccharomyces cerevisiae antibodies (ASCA) are a specific but only moderately sensitive diagnostic marker for Crohn's disease. We sought to explore the role of ASCA as a prognostic marker for aggressive disease phenotype in Crohn's disease.

Aims: To determine the role of ASCA status as a risk factor for early surgery in Crohn's disease. Subjects: We performed a case control study in a cohort of patients, newly diagnosed with Crohn's disease, between 1991 and 1999. All patients were followed for at least three years. Case subjects $(n=35)$ included those who had major surgery for Crohn's disease within three years of diagnosis. Controls $(n=35)$ included patients matched to cases for age, sex, disease location, and smoking status, and who did not undergo major surgery for Crohn's disease within three years of diagnosis.

Methods: Blinded assays were performed on serum for ASCA (immunoglobulin (lg)A and $\lg G$ ). A paired analysis of cases-controls was performed to test for the association between ASCA status and risk of early surgery.

Results: ASCA IgA was strongly associated with early surgery (odds ratio (OR) 8.5 (95\% confidence interval (CI) 2.0-75.9); $p=0.0013)$. ASCA $\lg G+$ and ASCA $\lg G+/ \lg A+$ patients were also at increased risk for early surgery (OR $5.5(95 \% \mathrm{Cl} 1.2-51.1), p=0.0265$; and $\mathrm{OR} 5.0(95 \% \mathrm{Cl} 1.1-46.9)$, $p=0.0433$, respectively). The association between ASCA and early surgery was evident in patients requiring surgery for ileal or ileocolonic disease.

Conclusions: Patients with Crohn's disease who are positive for ASCA lgA, lgG, or both, may define a subset of patients with Crohn's disease at increased risk for early surgery.
$M$ edical therapy for Crohn's disease (CD) is not curative, and relies on a variety of approaches to suppress bowel inflammation and the mucosal immune response. As reliable indicators of prognosis are lacking, medical therapy is usually guided by signs and symptoms. Treatment usually progresses sequentially through less effective therapies with few side effects, such as 5-aminosalicylates and antibiotics, to more efficacious therapies that may be associated with increased risk of serious adverse events, such as corticosteroids, immunomodulators, and anti-tumour necrosis factor (TNF) treatments. ${ }^{1}$

Despite this empiric approach to medical therapy, published data indicate that approximately $80 \%$ of CD patients require surgery by 20 years ${ }^{2}$ while $20-40 \%$ require their first surgery within three years of diagnosis. ${ }^{2-5}$ As resection is not curative, surgery is usually reserved for medically refractory disease, or for individuals with inflammatory sequelae such as stricture, fistula, or abscess. It is not known whether a more aggressive approach to medical therapy, such as treatment with immunomodulators or anti-TNF antibodies given soon after diagnosis, might alter the course of disease and decrease the rate of surgery. A limiting factor in such an approach is the poor ability to identify at the time of diagnosis those patients with an unfavourable prognosis. Given the increased risk of side effects associated with these medications, the risk-benefit analysis of this alternative approach would likely favour its application to the subgroup of patients at increased risk for poorer prognosis.

In 1988, Main et al observed that serum titres of both immunoglobulin (Ig)A and IgG antibodies against Saccharomyces cerevisiae (ASCA) were higher among patients with CD compared with controls. ${ }^{6}$ Approximately $60 \%$ of CD patients may be found to have ASCA present..$^{6-9}$ Despite this modest sensitivity, several studies have found ASCA expression (either IgA or IgG) to be nearly 95\% specific for CD. ${ }^{8} 1011$

In addition to its utility as a diagnostic marker for CD, more recent evidence suggests that ASCA serology may also correlate with disease behaviour. ASCA titres have been shown to be positively associated with early age of disease onset, fibrostenosis, and internal fistulas. ${ }^{12}{ }^{13}$ Additional evidence suggests an association of ASCA with disease location, with a reported linkage to ileal involvement. ${ }^{13}$ Furthermore, among patients with CD with ileal involvement, ASCA has been associated with a higher incidence of small bowel surgery. ${ }^{12} 13$

In a cohort of patients in New England diagnosed with CD, we have found that $20 \%$ underwent early surgery (defined as occurring within three years of diagnosis, exclusive of surgery that was simultaneous with diagnosis). ${ }^{14}$ Baseline clinical characteristics independently associated with early surgery were disease location (decreased risk associated with isolated colonic localisation) and cigarette smoking (increased risk). The aim of this study was to determine if ASCA serological status is associated with increased risk for early surgery.

\section{METHODS}

\section{Study population}

A consortium of 16 investigative sites in New England (referral centres and community based physicians) characterised 345 patients diagnosed with CD between 1991 and

Abbreviations: ASCA, anti-Saccharomyces cerevisiae antibodies; pANCA, perinuclear antineutrophil cytoplasmic antibodies; OmpC, Escherichia coli outer membrane porin C; CD, Crohn's disease; OR, odds ratio; TNF, tumour necrosis factor; Ig, immunoglobulin 
1999 and followed for at least three years. ${ }^{14}$ Diagnosis was confirmed according to standard radiographic, endoscopic, and histological criteria. Retrospective data collection was performed on baseline characteristics, medication use in the first three years, and incidence of surgery within three years of diagnosis. ${ }^{14}$

Among this cohort of 345 patients, 69 required major surgery within three years of diagnosis, excluding surgery performed at diagnosis. Major surgery was defined as any intra-abdominal surgical procedure, complex abscess drainage, or complex perianal fistula surgery performed for CD. Isolated incision and drainage of perianal abscess and simple perianal fistulectomy did not qualify as surgery in this outcome definition.

Written informed consent was obtained to collect blood for serology from 35 such "cases" (major surgery within three years of diagnosis, exclusive of surgery performed at diagnosis) from this cohort. Blood was drawn from 30 "controls" (no major surgery within three years of diagnosis) who were matched to cases on the basis of sex, age \pm 7 years, location of disease, and smoking behaviour at the time of diagnosis. An additional five control subjects were enrolled from the personal practice of one of the authors (BES) when no match could be found within the cohort of 345 patients.

A sample of venous blood was drawn from each paired subject. Serum was separated by centrifugation and stored at $-80^{\circ} \mathrm{C}$. Blinded samples were sent to Prometheus Laboratories (San Diego, California, USA) for ASCA (IgA and IgG), DNAse sensitive perinuclear antineutrophil cytoplasmic antibody (pANCA), and anti-OmpC (Escherichia coli outer membrane porin C) Ig A testing.

\section{Serological assays}

ASCA

Standard ELISA assays were performed using an oligosaccharide mannan preparation derived from Saccharomyces uvarum. Results were reported as EU, a ratio of sample OD to that obtained with the standard. An ASCA IgA ELISA of greater than $20.0 \mathrm{EU} / \mathrm{ml}$ and an IgG ELISA of greater than $40.0 \mathrm{EU} / \mathrm{ml}$ were considered positive test results.

\section{DNAse sensitive pANCA}

Patient serum samples were screened for the presence of IgG anti-ANCA using an ELISA format with Ficoll-Hypaque gradient purified human neutrophils. Serum samples were applied to PMN coated slides and the presence of a peripheral pattern of staining was determined by immunofluorescence microscopy. Sera which were considered to be positive for DNase sensitive pANCA were positive on both ELISA screening and in addition were observed to have a DNAse sensitive immunofluorescence pattern.

\section{Anti-OmpC}

ELISA wells were coated with purified OmpC antigen. Patient samples, serum standard, and low and high serum controls were diluted 1:100 and added to the wells. After incubation and wash steps, the presence of bound human IgA antibodies was demonstrated with goat antihuman IgA-alkaline phosphatase antibody. An anti-OmpC IgA ELISA of greater than 16.5 EU/ml was considered a positive test result.

\section{Statistical analysis}

A two tailed McNemar's test was used to compare paired proportions (matched cases and controls) of the frequency of positive serum values for ASCA, pANCA, and anti-OmpC. Results are reported as odds ratio (OR) and 95\% confidence interval (CI), with p values. Statistically significant findings were identified at an alpha level of $p \leqslant 0.05$. Correlations between ASCA titre and time from diagnosis to serology and between ASCA titre and time from surgery to serology were explored using Pearson's correlation coefficient. Two tailed Fisher's exact tests were used to obtain nominal $p$ values for exploratory comparisons of baseline demographic and clinical features of the groups of patients comprising cases and controls. Exploratory analyses of the association of ASCA titre and risk of early surgery were performed using a Wilcoxon rank sum test. Cochran-Mantel-Haenszel tests were used to perform a stratified analysis of the risk of early surgery in association with ASCA while controlling for the presence or absence of pANCA and OmpC. The study was

Table 1 Patient demographic and clinical features

\begin{tabular}{|c|c|c|c|}
\hline & $\begin{array}{l}\text { Early surgery } \\
(n=35)\end{array}$ & $\begin{array}{l}\text { No early surgery } \\
(n=35)\end{array}$ & $\begin{array}{l}\text { Nominal } \\
\text { p value }\end{array}$ \\
\hline Age at diagnosis ${ }^{*}(y)$ (mean (range)) & $32(22-74)$ & $32(23-73)$ & 1.000 \\
\hline \multicolumn{4}{|l|}{$\operatorname{Sex}^{*}($ No $(\%))$} \\
\hline Female & $20(57.1)$ & $20(58.3)$ & 1.000 \\
\hline Male & $15(42.9)$ & $15(42.9)$ & 1.000 \\
\hline \multicolumn{4}{|l|}{ Disease location* (No (\%)) } \\
\hline lleum only & $11(31.4)$ & $11(31.4)$ & 1.000 \\
\hline Colon only & $10(28.6)$ & $10(28.6)$ & 1.000 \\
\hline lleum and colon & $14(40.0)$ & $14(40.0)$ & 1.000 \\
\hline \multicolumn{4}{|l|}{ Smoking status at diagnosis* (No (\%)) } \\
\hline Smoker & $7(20.0)$ & $7(20.0)$ & 1.000 \\
\hline Non-smoker & $17(48.6)$ & $17(48.6)$ & 1.000 \\
\hline Unknown & $11(31.4)$ & $11(31.4)$ & 1.000 \\
\hline Mean time to diagnosis from onset of symptoms (days) & 589 & 404 & 0.367 \\
\hline \multicolumn{4}{|l|}{ Medication use in the first three years (No (\%)) } \\
\hline 5-ASA & $32(91.4)$ & $23(65.7)$ & 0.010 \\
\hline Oral steroids & $29(82.9)$ & $24(68.6)$ & 0.264 \\
\hline IV steroids & $7(20.0)$ & $6(17.2)$ & 0.760 \\
\hline 6-MP or azathioprine & $18(51.4)$ & $13(37.1)$ & 0.242 \\
\hline Methotrexate & $3(8.6)$ & $3(8.6)$ & 1.000 \\
\hline Cyclosporin & $0(0.0)$ & $1(2.9)$ & 0.500 \\
\hline Infliximab & $0(0.0)$ & $2(5.7)$ & 0.247 \\
\hline Presence of extraintestinal manifestations (No (\%)) & $9(25.7)$ & $6(17.1)$ & 0.402 \\
\hline \multicolumn{4}{|l|}{ Practice where treated (No (\%)) } \\
\hline Community & $12(34.3)$ & $9(25.7)$ & 0.450 \\
\hline Referral & $23(65.7)$ & $26(74.3)$ & 0.450 \\
\hline
\end{tabular}


Table 2 Features of patients who underwent surgery within three years of diagnosis

\begin{tabular}{ll}
\hline & Cases $(\mathbf{n}=35)$ \\
\hline Type of surgical procedure (No (\%)) & \\
Ileocaecal resection & $23(65.8)$ \\
Complex abscess drainage & $2(5.7)$ \\
Complex perianal surgery & $6(17.1)$ \\
Colon resection & $4(11.4)$ \\
Indication for surgery (No (\%)) & \\
Stricture & $8(22.9)$ \\
Intra-abdominal abscess & $5(17.1)$ \\
Pelvic abscess & $1(2.9)$ \\
Perianal abscess & $2(5.7)$ \\
Perianal fistula & $4(11.4)$ \\
Enteroenteric fistula & $4(11.4)$ \\
Rectovaginal fistula & $1(2.9)$ \\
Enterovesical fistula & $1(2.9)$ \\
Refractory to medical treatment & $7(20.0)$ \\
Time from diagnosis to surgery (No (\%)) & $17(47.2)$ \\
$>6$ months & $18(51.4)$ \\
6 months-3 years &
\end{tabular}

approved by the Institutional Review Board at the Massachusetts General Hospital.

\section{RESULTS}

Of the 70 patients in the paired analysis, the only significant difference between the case and control populations was with regard to use of aminosalicylate medications in the first three years. Use of an aminosalicylate preparation within three years of diagnosis was associated with early surgery $(\mathrm{p}=0.010)$. There were no significant differences with regard to time from onset of symptoms to diagnosis, type of practice in which the patient received his or her care, presence of extraintestinal manifestations, or use of other medications (table 1 ).

Among the 35 patients who underwent major surgery within three years of diagnosis with $\mathrm{CD}$, the most common surgery performed was an ileocaecal resection (table 2 ). The most common indications for surgery were clinically important strictures and refractoriness to medical therapy. Nearly equal numbers of patients underwent surgery within six months of diagnosis and from six months to three years.

Of the 70 patients studied, $43 \%$ were ASCA positive (either IgA or IgG). Specifically, $41 \%$ were positive for ASCA IgA, $27 \%$ for ASCA IgG, and $26 \% \%$ for ASCA IgA and IgG (double positive) (table 3 ). Overall, $21 \%$ of patients were positive for DNAse sensitive pANCA and 20\% for anti-OmpC.

ASCA IgA positivity was strongly associated with early surgery (OR 8.5 (95\% CI 2.0-75.9); $\mathrm{p}=0.0013)$. ASCA IgG+ and ASCA IgG+/IgA+ patients were also at increased risk for early surgery (OR 5.5 (95\% CI 1.2-51.1), p = 0.0265; and OR
Table 4 Serological profiles of individual patients

\begin{tabular}{lll}
\hline Serology cluster* & $\begin{array}{l}\text { Early surgery } \\
(\mathbf{n}=\mathbf{3 5})\end{array}$ & $\begin{array}{l}\text { No early surgery } \\
(\mathbf{n}=\mathbf{3 5})\end{array}$ \\
\hline ASCA-/pANCA-/OmpC- & 10 & 14 \\
ASCA-/pANCA-/OmpC+ & 1 & 4 \\
ASCA-/pANCA+/OmpC- & 1 & 7 \\
ASCA-/pANCA+/OmpC+ & 0 & 3 \\
ASCA+/pANCA-/OmpC- & 16 & 4 \\
ASCA+/pANCA-/OmpC+ & 3 & 3 \\
ASCA+/pANCA+/OmpC- & 4 & 0 \\
ASCA+/pANCA+/OmpC+ & 0 & 0
\end{tabular}

ASCA, anti-Saccharomyces cerevisiae antibodies; pANCA, perinuclear antineutrophil cytoplasmic antibodies; OmpC, Escherichia coli outer membrane porin $\mathrm{C} ; \mathrm{lg}$, immunoglobulin.

${ }^{*} p=0.0003$ for association of ASCA ( $\lg A$ or $\left.\lg G\right)$ and early surgery, controlling for $\mathrm{pANCA}$ and $\mathrm{OmpC}$.

$p=0.082$ for association of OmpC and early surgery, controlling for ASCA (lgA or $\lg G)$ and pANCA.

$p=0.32$ for association of pANCA and early surgery, controlling for ASCA (lgA or $\lg G)$ and OmpC.

5.0 (95\% CI 1.1-46.9), p = 0.0433, respectively). Neither antiOmpC nor DNase sensitive pANCA status were associated with early surgery (table 3 ).

We also analysed our data to explore potential associations of ASCA with regard to type of surgery needed. This analysis demonstrated that the strong association between ASCA IgA status and surgery was greatest among those with ileocaecal resection and complex intra-abdominal abscess drainage (number of pairs $=25$ ), with an OR of 13.0 (95\% CI $1.95-$ $552 ; p=0.0033)$. Separate analysis for the remaining cases who underwent colon resection or complex perianal surgery (number of pairs $=10$ ) did not demonstrate a statistically significant association, with an OR of 3.0 (95\% CI 0.241-157; $\mathrm{p}=0.6171)$.

We explored whether distinct combinations of serologies were associated with early surgery (table 4 ). When controlling for the presence of pANCA and OmpC, ASCA positivity (either IgA or IgG) was found to be associated with the risk for early surgery $(p=0.0003)$. There was a trend for association of early surgery and OmpC positivity, controlling for pANCA and ASCA $(\mathrm{p}=0.082)$. pANCA was not associated with early surgery when controlling for OmpC and ASCA $(\mathrm{p}=0.32)$.

We did not observe a correlation between ASCA IgA titre and time from surgery to blood draw for serology $(r=-0.0236, \mathrm{p}=0.891)$ or from diagnosis of CD to blood draw for serology $(r=0.0127, \mathrm{p}=0.942)$.

Higher mean antibody titres (ASCA IgA and $\operatorname{IgG}$ ) were noted among patients who had undergone early surgery (table 5). Mean titres were $38.3 \mathrm{EU} / \mathrm{ml}$ and $15.4 \mathrm{EU} / \mathrm{ml}$ for ASCA IgA (early surgery and no early surgery, respectively), and $44.0 \mathrm{EU} / \mathrm{ml}$ and $22.7 \mathrm{EU} / \mathrm{ml}$ for ASCA IgG $(p=0.0004$ for IgA and 0.0009 for $\operatorname{IgG})$. However, a large number of

Table 3 Frequency of positive serum values for ASCA, pANCA, and OmpC, and results of McNemar's test

\begin{tabular}{llllll}
\hline & $\begin{array}{l}\text { All subjects } \\
(\mathbf{n}=\mathbf{7 0})\end{array}$ & $\begin{array}{l}\text { Early surgery } \\
(\mathbf{n}=\mathbf{3 5 )}\end{array}$ & $\begin{array}{l}\text { No early surgery } \\
(\mathbf{n}=\mathbf{3 5})\end{array}$ & $\mathbf{p}$ Value & OR (95\% Cl) \\
\hline ASCA & & & & & \\
ASCA IgA (No (\%)) & $29(41.4)$ & $22(62.9)$ & $7(20.0)$ & $0.001^{*}$ & $8.50(2.02-75.85)$ \\
ASCA lgG (No (\%)) & $19(27.1)$ & $14(40.0)$ & $5(14.3)$ & $0.027^{*}$ & $5.50(1.20-51.07)$ \\
ASCA lgA and lgG (No (\%)) & $18(25.7)$ & $13(37.1)$ & $5(14.3)$ & $0.043^{*}$ & $5.00(1.07-46.93)$ \\
DNAse sensitive pANCA (No (\%)) & $15(21.4)$ & $5(14.3)$ & $10(28.6)$ & 0.423 & $0.56(0.15-1.85)$ \\
OmpC (No (\%)) & $14(20.0)$ & $4(11.4)$ & $10(28.6)$ & 0.114 & $0.250(0.03-1.25)$ \\
\hline
\end{tabular}

*Statistically significant.

ASCA, anti-Saccharomyces cerevisiae antibodies; pANCA, perinuclear antineutrophil cytoplasmic antibodies; OmpC, Escherichia coli outer membrane porin C; Ig, immunoglobulin.

OR ( $95 \% \mathrm{Cl})$, odds ratio (95\% confidence interval). 
Table 5 ASCA serology titres with respect to time of surgery

\begin{tabular}{llll}
\hline & $\begin{array}{l}\text { Early surgery } \\
(\mathbf{n}=35)\end{array}$ & $\begin{array}{l}\text { No early surgery } \\
(\mathbf{n = 3 5})\end{array}$ & p Value \\
\hline ASCA lgA positive (No) & 22 & 7 & - \\
Titre range (EU/ml) & $0-111.7$ & $0-114.1$ & - \\
Mean titre (all patients) (EU/ml) & 38.3 & 15.3 & $0.0004^{*}$ \\
Mean titre (positive patients only) (EU/ml) & 49.6 & 48.6 & 0.872 \\
ASCA lgG positive (No) & 14 & 5 & - \\
Titre range (EU/ml) & $0-171.0$ & $0-191.3$ & - \\
Mean (all patients) (EU/ml) & 44.0 & 22.6 & $0.0009^{*}$ \\
Mean titre (positive patients only) (EU/ml) & 51.4 & 46.6 & \\
\hline ASCA, anti-Saccharomyces cerevisiae antibodies; lg, immunoglobulin. & & \\
*Statistically significant. &
\end{tabular}

individuals had values for ASCA IgA and IgG of "0.0" and to further characterise the relationship between ASCA titre and risk of early surgery, we performed additional analyses excluding patients with a titre of " 0.0 ". Stratifying in this way, we noted a mean titre of $49.6 \mathrm{EU} / \mathrm{ml}$ and $48.6 \mathrm{EU} / \mathrm{ml}$ for IgA (early surgery and no early surgery, respectively), and $51.4 \mathrm{EU} / \mathrm{ml}$ and $46.6 \mathrm{EU} / \mathrm{ml}$ for ASCA $\operatorname{IgG}(\mathrm{p}=0.87$ for $\operatorname{IgA}$ and 0.28 for $\operatorname{IgG}$ ). These data indicate that higher titres of ASCA IgA or IgG antibodies do not correlate with the risk for early surgery better than meeting the definition of positive or negative ASCA serology alone. Similar mean antibody titres (ASCA IgA and IgG) were noted among patients who had received their care in referral centres or from community based physicians (table 6).

\section{DISCUSSION}

In this study, we have demonstrated that patients with CD who are positive for ASCA IgA, IgG, or both, have a significantly increased risk of having had early surgery (within three years of diagnosis). While controlling for age, sex, smoking status, and disease location by matching on these characteristics, ASCA IgA positivity was strongly associated with early surgery, with an OR of 8.50. Although our study was small, the paired analysis used in this case control study enhanced our ability to discern this association. Our study is the first to identify ASCA status as a strong independent risk factor for early surgery in patients with CD.

In addition, in agreement with the findings of Vasiliauskas and colleagues, ${ }^{12}$ we observed that the association between ASCA positivity and early surgery exists for patients who had surgery for complications of ileal or ileocolonic disease, above and beyond the risk associated with this disease localisation itself. Increased risk of surgery in association with ASCA positivity could not be detected for those who needed surgery for complications of colonic disease (the latter also being strongly associated with perianal disease). While it is possible that the numbers of patients with purely colonic disease were too small to have detected an association, our data support the hypothesis that patients who are ASCA positive are at a greatly increased risk for early surgery compared with patients with the same disease location who are ASCA negative, particularly with ileal or ileocolonic disease.
Other serological markers of inflammatory bowel disease, namely DNase sensitive pANCA and anti-OmpC, were not associated with early surgery. Antibodies to the Escherichia coli outer membrane porin C (IgA anti-OmpC) have been noted in $55 \%$ of patients with $\mathrm{CD} .{ }^{15}$ In contrast, DNase sensitive pANCA is more strongly associated with ulcerative colitis than $C D$, and has been shown to be associated with certain disease behaviours in CD. Approximately $10 \%$ of CD patients are DNase sensitive pANCA positive. Several groups have shown that DNase sensitive pANCA is associated with symptomatic and endoscopically apparent left sided colitis when positive in CD. ${ }^{11}{ }^{16-18}$ Although we found no association between pANCA or OmpC and early surgery, the small numbers of patients studied and low proportion of patients positive for these serologies prevents us from reaching any definitive conclusions about their role as risk factors for early surgery.

Limited data are available regarding the stability of these serum markers over time, and in relation to fluctuations of disease activity, and to medical or surgical treatments. Landers et al looked at titres of ASCA, DNase sensitive pANCA, and anti-OmpC in a cohort of patients with CD before and after treatment with infliximab. ${ }^{15}$ No significant correlation between serum marker titres and changes in disease activity was found. ${ }^{15}$ Ruemmele et al found that among paediatric patients with $\mathrm{CD}$ who had undergone surgery, ASCA titres drifted towards normal levels postoperatively. ${ }^{11}$ Teml et al found ASCA titres to be stable over time with mesalamine treatment, and had a tendency to decrease with corticosteroid treatment. ${ }^{19}$ As we measured serological titres after surgery had occurred in our cases, the main concern for confounding of the association we observed between early surgery and ASCA positivity would be if one were to see titres decreasing after surgery. However, such a tendency, if present, would bias our findings towards a lack of association, and would have weakened the observed effect size. In this regard, we did not observe any correlation of ASCA IgA titre with either of two intervals: time between surgery and blood draw for ASCA titre or time from diagnosis of CD to measure of ASCA titre. Although our analysis was limited by not having baseline ASCA titre levels (that is, drawn at the time of diagnosis), our data support the notion

Table 6 ASCA serology titres with respect to type of practice in which patients received their care

\begin{tabular}{llll}
\hline & $\begin{array}{l}\text { Community practice } \\
(\mathbf{n}=\mathbf{2 1})\end{array}$ & $\begin{array}{l}\text { Referral practice } \\
(\mathbf{n}=49)\end{array}$ & $\mathbf{p}$ Value \\
\hline ASCA IgA mean titre & 27.1 & 26.6 & 0.756 \\
ASCA IgG mean titre & 39.1 & 31.2 & 0.463 \\
\hline ASCA, anti-Saccharomyces cerevisiae antibodies; Ig, immunoglobulin. &
\end{tabular}


that ASCA IgA titres remain stable over time and are unaffected by surgical therapy itself. In addition, our data indicate that the threshold effect of being positive or negative for ASCA, rather than the actual titre, fully accounts for the association between ASCA and early surgery.

We have previously shown that smoking and isolated colonic disease localisation are independent risk factors for early surgery, with OR values of 3.09 and 0.27 , respectively. ${ }^{14}$ Having used case control methodology to match our patients on both disease location and smoking status at diagnosis, our analysis eliminates any potential confounding effect of these independent risk factors for early surgery in relation to serological status. The present study provides strong evidence that ASCA status is an additional risk factor for early surgery in patients with $\mathrm{CD}$.

None the less, our study has several limitations. The retrospective nature of the study introduces the potential for bias in data collection. In this regard, it is important to note that cases and controls were matched with no knowledge of ASCA status. A second limitation is that most of the subjects enrolled in this study were managed before the era of antiTNF therapy. Therefore, the potential impact of infliximab treatment on the outcome of interest (early surgery) cannot be discerned.

It is of interest that a negative association has been reported between ASCA and smoking. ${ }^{20}$ In evaluating our own cohort, we did not observe any difference in ASCA status between non-smokers and smokers $(p=0.753)$; however, numbers were small. A more precise definition of smoking behaviours in our patient populations may have been desirable. Cosnes et al detected a threshold effect at 15 cigarettes per day on the severity of $\mathrm{CD}^{21}$ In our study, smoking behaviour was defined as smoking status at the time of diagnosis. However, as these data were collected retrospectively, it was not possible to reliably ascertain the precise number of cigarettes smoked. Having matched cases and controls on the basis of broad categories of smoking behaviour, we believe we have minimised the effect of smoking as a potential confounder on the outcome of early surgery. For the threshold effect to be of importance as a confounder despite our having matched on smoking behaviour, one would need to assume two conditions: (a) that more cases who were smokers smoked more numbers of cigarettes than controls who were smokers; and (b) that number of cigarettes smoked was also positively associated with ASCA positivity. As the existing evidence suggests an inverse correlation between smoking and ASCA positivity (that is, smokers are less likely to be ASCA positive), ${ }^{20}$ we believe that more detailed data on smoking exposure, although potentially of interest in refining our analysis, would have been unlikely to controvert our findings. It is still possible that other factors yet to be identified may be confounding the association identified between ASCA and early surgery.

It has also been suggested that disease behaviour, defined according to the Vienna classification as stricturing, penetrating, or neither, ${ }^{22}$ might also predict risk for surgery. The original data collection of the retrospective cohort from which our study was derived was to have recorded clinical and laboratory data identified at the time of diagnosis. Given the focus of the original study, we did not record data from studies performed after diagnosis. The cohort did include $5.4 \%$ of patients who had perianal fistula at the time of diagnosis but these patients did not have an increased risk of early surgery. ${ }^{14}$ Furthermore, it appears that the Vienna classification behaviour characteristics tend to evolve over the course of follow up and do not appear to be completely independent of disease location, ${ }^{23}$ perhaps diminishing the utility of disease behaviour as a predictive factor.
Identifying individuals at high risk for early surgery at the time of diagnosis in CD might enable clinicians to develop new treatment algorithms aimed at forestalling the natural progression of the disease and its complications. The current CD management paradigm is based on a "step up" approach, in which there is an escalation of medication potency over time as the disease progresses. In this algorithm, 5 -aminosalicylate preparations are often used as first line agents, followed by corticosteroids, immune modulators (6mercaptopurine, azathioprine, methotrexate), and more recently, infliximab.

At present, it is not known if the natural history of CD would be modified if the usual "step up" treatment paradigm were reversed ("step down"), such that immune modulators and biological therapies were instituted earlier in the disease process. On the basis of this study, one may hypothesise that ASCA status might define a subset of patients with CD who are at sufficiently high risk for early surgery to merit initiating therapy with an immunomodulatory agent soon after diagnosis. Prospective studies are warranted to further characterise the role of ASCA status and other clinical and subclinical characteristics in predicting clinical outcomes.

\section{ACKNOWLEDGEMENTS}

Dr Sands is supported by a Mentored Patient-Oriented Research Award from the National Institutes of Health, 5 K23 DK02850. Dr Forcione is supported by an NIH Training Grant, DK 07191. Dr Rosen was supported by the PASTEUR program at Harvard Medical School. $\mathrm{Mr}$ Kisiel was supported by a Doris Duke Clinical Research Fellowship, and participated in the PASTEUR program at Harvard Medical School, Boston, MA. Dr Sands has been a consultant for Prometheus Laboratories (San Diego, California, USA). Serological assays were provided by Prometheus Laboratories. The authors wish to thank Mr Jason Do of Prometheus Laboratories for facilitation of serological assays and comments on this manuscript, and Elkan Halpern, PhD, for biostatistical support

\section{Authors' affiliations}

D G Forcione, M J Rosen, J B Kisiel, B E Sands, Gastrointestinal Unit and Center for the Study of Inflammatory Bowel Disease, Massachusetts General Hospital and Harvard Medical School, Boston, MA, USA

\section{REFERENCES}

1 Hananuer SB, Sandborn WJ. Management of Crohn's disease in adults. Am J Gastroenterol 2001 ; 96:635-43.

2 Mekhiian HS, Switz DM, Melnyk CS, et al. Clinical features and natural history of Crohn's disease. Gastroenterology 1979;77:898-906.

3 Binder V, Hendriksen C, Kreiner S. Prognosis in Crohn's disease-based on results from a regional patient group from the county of Copenhagen. Gut 1985:26:146-50.

4 Elliott PR, Ritchie JK, Lennard-Jones JE. Prognosis of colonic Crohn's disease. Br Med J (Clin Res Ed) 1985;291:178.

5 Basilisco G, Campanini M, Cesana B, et al. Risk factors for first operation in Crohn's disease. Am J Gastroenterol 1989:84:749-52.

6 Main J, McKenzie H, Yeaman GR, et al. Antibody to Saccharomyces cerevisiae (bakers' yeast) in Crohn's disease. BMJ 1988;297:1 105-6.

7 Sendid B, Colombel JF, Jacquinot PM, et al. Specific antibody response to oligomannosidic epitopes in Crohn's disease. Clin Diagn Lab Immunol 1996;3:219-26.

8 Quinton JF, Sendid B, Reumaux D, et al. Anti-Saccharomyces cerevisiae mannan antibodies combined with antineutrophil cytoplasmic autoantibodies in inflammatory bowel disease: prevalence and diagnostic role. Gut 1998;42:788-91.

9 Peeters M, Joossens S, Vermeire S, et al. Diagnostic value of antiSaccharomyces cerevisiae and antineutrophil cytoplasmic autoantibodies in inflammatory bowel disease. Am J Gastroenterol 2001;96:730-4.

10 Hoffenberg EJ, Fidanza S, Sauaia A. Serologic testing for inflammatory bowel disease. J Pediatr 1999;134:447-52.

11 Ruemmele FM, Targan SR, Levy G, et al. Diagnostic accuracy of serological assays in pediatric inflammatory bowel disease. Gastroenterology 1998:115:822-9.

12 Vasiliauskas EA, Kam LY, Karp LC, et al. Marker antibody expression stratifies Crohn's disease into immunologically homogeneous subgroups with distinct clinical characteristics. Gut 2000:47:487-96.

13 Sostegni R, Daperno M, Ercole E, et al. A. Detection of anti-Saccharomyces cerevisiae antibodies in Crohn's disease: is it a reliable diagnostic and prognostic marker, Dig Liver Dis 2001;33:755-61. 
14 Sands BE, Arsenault JE, Rosen MJ, et al. Risk of early surgery for Crohn's disease: Implications for early treatment strategies. Am J Gastroenterol 2003;98:2712-18.

15 Landers CJ, Cohavy O, Misra R, et al. Selected loss of tolerance evidenced by Crohn's disease-associated immune responses to auto- and microbial antigens. Gastroenterology 2002;123:689-99.

16 Vasiliauskas EA, Plevy SE, Landers CJ, et al. Perinuclear antineutrophil cytoplasmic antibodies in patients with Crohn's disease define a clinica subgroup. Gastroenterology 1996;110:1810-19.

17 Satsangi J, Landers CJ, Welsh KI, et al. The presence of anti-neutrophil antibodies reflects clinical and genetic heterogeneity within inflammatory bowel disease. Inflamm Bowel Dis 1998;4:18-26.

18 Freeman $\mathrm{H}$. Atypical perinuclear antineutrophil cytoplasmic antibodies (p-ANCA) in patients with Crohn's disease. Gastroenterology 1998;114:A979.
19 Teml A Kratzer V Schneider B, et al. Anti-Saccharomyces cerevisiae antibodies: a stable marker for Crohn's disease during steroid and 5aminosalicylic acid treatment. Am J Gastroenterol 2003;98:2226-31.

20 van Kemseke C, Belaiche J, Steeman C, et al. Negative association between smoking and anti-saccharomyces cerevisiae antibodies in Crohn's disease. Acta Gastroenterologica Begica 2003;66:1-6.

21 Cosnes J, Carbonnel F, Carrat F, et al. Effects of current and former cigarette smoking on the clinical course of Crohn's disease. Alimentary Pharm Ther 1999;13:1403-11.

22 Gasche C, Scholmerich J, Brynskov J, et al. A simple classification of Crohn's disease: report of the working party of the world congresses of gastroenterology, Vienna 1998. Inflamm Bowel Dis 2000;6:8-15.

23 Louis E, Collard A, Oger AF, et al. Behaviour of Crohn's disease according to the Vienna classification: changing pattern over the course of the disease. Gut 2001:49:777-82.

\section{EDITOR'S QUIZ: GI SNAPSHOT}

\section{A male with a pelvic mass}

\section{Clinical presentation}

A 40 year old man presented to the emergency department with a six day history of intermittent abdominal left lower quadrant pain, nausea, vomiting, and constipation. The patient admitted excessive fruit and vegetable consumption for the past six months. His past medical history was not revealing.

On physical examination the abdomen was moderately distended, soft, and with normal bowel sounds. A tender $8 \times 6 \mathrm{~cm}$ mass was palpable in the left lower quadrant. Digital rectal examination, laboratory tests, and chest $x$ ray (fig 1 ) were unremarkable.

\section{Question}

What does this plain abdominal $x$ ray show?

See page 1144 for answer

This case is submitted by:

K T Kjossev, N N Vladov Department of Surgery, Military Medical Academy, Sofia, Bulgaria J E Losanoff University of Missouri, Columbia, Missouri, USA

Correspondence to: Dr Kirien Kjossev, PO Box 159, Department of Surgery, Military Medical Academy, 1606 Sofia, Bulgaria; kirien@omega.bg



Figure 1 